\title{
Influence of CFH gene on symptom severity of schizophrenia
}

\author{
This article was published in the following Dove Press journal: \\ Neuropsychiatric Disease and Treatment \\ 2 March 2017 \\ Number of times this article has been viewed
}

\section{Chen Zhang' \\ Qinyu Lv' \\ Weixing Fan² \\ Wei Tang ${ }^{3}$ \\ Zhenghui Yi'}

'Schizophrenia Program, Shanghai Mental Health Center, Shanghai Jiao Tong University School of Medicine, Shanghai, ${ }^{2}$ Department of Psychiatry, Jinhua Second Hospital, Jinhua, ${ }^{3}$ Department of Psychiatry, Wenzhou Kanging Hospital, Wenzhou, People's Republic of China
Correspondence: Chen Zhang; Zhenghui Yi

Schizophrenia Program, Shanghai Mental Health Center, Shanghai Jiao Tong University School of Medicine, 600 Wan Ping Nan Road, Shanghai 200030, People's Republic of China Email zhangchen645@gmail.com; yizhenghuil97I@I63.com
Objective: Recent advances have provided compelling evidence for the role of excessive complement activity in the pathophysiology of schizophrenia. In this study, we aimed to detect the association of the gene encoding complement factor $\mathrm{H}(\mathrm{CFH})$, a regulator in complement activation, with schizophrenia.

Materials and methods: A sample of 1783 individuals with or without schizophrenia was recruited for genetic analysis. Genomic DNA samples were extracted from peripheral blood cells using multiplex polymerase chain reaction and the SNaPshot assay. A Database for Schizophrenia Genetic Research (SZDB) was used to detect the association of brain $\mathrm{CFH}$ expression with schizophrenia. Next, we performed a genotype-phenotype analysis to identify the relationship between $C F H$ Y $402 \mathrm{H}$ polymorphism and clinical features of schizophrenia.

Results: There was a significant association of hippocampal $C F H$ expression with schizophrenia $(P=0.017)$, whereas this significance did not survive after adjusting for false discovery rate $(P=0.105)$. Comparing the genotype and allele frequencies of the genotyped single-nucleotide polymorphisms between case and control groups showed no significant difference. There were significant differences in the scores of negative symptoms and delayed memory between the patients with $\mathrm{C}$ allele and those without $\mathrm{C}$ allele $(P<0.01$ and $P=0.04$ after Bonferroni correction, respectively). Furthermore, we observed a marginally significant association between the $\mathrm{Y} 402 \mathrm{H}$ polymorphism and $\mathrm{CFH}$ expression in the hippocampus ( $P=0.051)$; however, this significance was lost after multiple testing correction ( $P=0.51$, after Bonferroni correction).

Conclusion: Our findings provide suggestive evidence for the role of $\mathrm{CFH}$ in the development of negative symptoms and cognitive dysfunction in schizophrenia.

Keywords: complement factor $\mathrm{H}$, negative symptoms, cognitive dysfunction, hippocampus, schizophrenia

\section{Introduction}

Schizophrenia is a chronic, severe and devastating neuropsychiatric disorder with a lifetime risk of $\sim 1 \%$ and characterized by positive and negative symptoms and cognitive dysfunction. Although intensive research has been done in the past decades, the biological mechanism of schizophrenia remains obscure. ${ }^{1}$ Early literature indicated that both maternal bacterial and viral infections during pregnancy epidemiologically increase the risk of schizophrenia in offspring. ${ }^{2}$ There is also evidence showing that patients with schizophrenia or certain autoimmune diseases share some key clinical, epidemiological and genetic features. ${ }^{3}$ Therefore, it is believed today that immune alterations may be involved in the pathophysiology of schizophrenia.

Family, twin and adoption studies have demonstrated that schizophrenia is a familiar disorder with a complex mode of inheritance, and its heritability reaches upward of $80 \% .{ }^{4,5}$ Hence, understanding the genetics involved in schizophrenia seems to provide 
a way to dissect the biological mechanism of this disorder. ${ }^{1}$ Recent genome-wide association studies (GWASs) have identified several genes within the extended human major histocompatibility complex (MHC) region conferring susceptibility to schizophrenia across different ethnics. ${ }^{6-11}$ Given the best role of MHC in immunity, Sekar et $\mathrm{al}^{12}$ reported a novel susceptibility gene encoding complement component 4 (C4) in schizophrenia, implying that excessive complement activity increases schizophrenia risk. In the activation of the complement system, complement factor $\mathrm{H}(\mathrm{CFH})$ acts as a major inhibitor of the alternative pathway in the complement cascade. ${ }^{13}$ Abnormalities in the structure or function of $\mathrm{CFH}$ can accordingly unbalance the normal homeostasis of the complement system, resulting in "bystander" damage to healthy tissues. Our previous work has reported that the gene encoding $\mathrm{CFH}(\mathrm{CFH})$ increases the risk for major depressive disorder (MDD) in Han Chinese. ${ }^{14}$ In clinics, patients with schizophrenia or MDD might share some symptoms such as loss of interests, sad mood, insomnia, energy and cognitive dysfunction. ${ }^{15,16}$ In genetics, there are quite a few studies indicating that both diseases might share some polygenic basis. ${ }^{17}$ Collectively, this study aimed to verify whether $\mathrm{CFH}$ has some potential associations with schizophrenia in Han Chinese.

Here, we first used a public database to detect whether $\mathrm{CFH}$ is differentially expressed in brain between patients with schizophrenia and healthy controls. Then, we genotyped a total of 11 single-nucleotide polymorphisms (SNPs), which were screened for a good coverage of this region in DNA samples of 1783 individuals with or without schizophrenia, in order to characterize the association between genetic variations within $\mathrm{CFH}$ and the risk of developing schizophrenia in Han Chinese. It has been well established that schizophrenia is a heterogeneous disease and clinical phenotype would be more close to certain susceptibility genes rather than the whole spectrum of schizophrenia. Hence, investigating the genotype-phenotype correlations of schizophrenia may lead to a more detailed understanding of this disease. ${ }^{18,19}$ A nonsynonymous SNP rs1061170 (Y402H) was reported to have a significant association with $\mathrm{MDD},{ }^{14}$ and we hypothesized that this functional polymorphism may have a genotypephenotype correlation with schizophrenia symptoms. In the third step, we analyzed the relationship between $\mathrm{Y} 402 \mathrm{H}$ and clinical features of schizophrenia.

\section{Materials and methods} Subjects

We recruited 878 patients with schizophrenia from three mental hospitals in Eastern China, including Shanghai Mental
Health Center, Shanghai Jiao Tong University School of Medicine, Jinhua Second Hospital and Wenzhou Kangning Hospital. All patients met the diagnoses of schizophrenia according to the Diagnostic and Statistical Manual of Mental Disorders, Fourth Edition (DSM-IV) and had no other observable physical disease or other psychiatric disorders aside from schizophrenia. Among them, there were 254 schizophrenia patients under olanzapine monotherapy enrolled for evaluating clinical features, whose inclusion criteria were according to our previous publications ${ }^{20-22}$ as follows: 1) duration of illness $<5$ years; 2) a minimum education of primary middle school; 3) receiving atypical antipsychotic monotherapy; 4) maintained a stable condition for $>6$ months before entry into the study and 5) a Positive and Negative Syndrome Scale for Schizophrenia (PANSS) total score $<60$.

A total of 905 healthy controls were recruited from hospital staff and students of School of Medicine in Shanghai and then interviewed by a specialized psychiatrist using the Structured Clinical Interview for DSM-IV-TR Axis I Disorders-Patient Edition.

All the patients and control subjects were of Han Chinese origin. All procedures were reviewed and approved by the institutional review boards of Shanghai Mental Health Center, Jinhua Second Hospital and Wenzhou Kanging Hospital. This study was performed in accordance with the guidelines laid out in the Declaration of Helsinki as revised in 1989. All subjects provided written informed consent before any study-related procedures were performed.

\section{Evaluation}

The PANSS was employed to evaluate symptom severity. ${ }^{22}$ The Repeatable Battery for the Assessment of Neuropsychological Status (RBANS) was the primary outcome instrument for this study. ${ }^{23}$ The 12-item RBANS consists of five subsets, corresponding to five domains of neuropsychological process: 1) immediate memory (list learning and story memory), 2) visuospatial/constructional (figure copy and line orientation), 3) language (picture naming and semantic fluency), 4) attention (digit span and coding) and 5) delayed memory (list learning free recall, list learning recognition, story memory free recall and figure free recall).

\section{Brain eQTL (expression quantitative trait loci) analysis for CFH expression}

It is known that schizophrenia originates from brain structural and functional abnormalities, ${ }^{24}$ and dysregulation of gene expression has a key role in the pathogenesis of this disease. In this study, we performed an eQTL analysis to detect 
whether $\mathrm{CFH}$ is differentially expressed in brain between patients with schizophrenia and healthy controls, using SZDB database (http://www.szdb.org/), a newly developed comprehensive resource for schizophrenia research. ${ }^{25}$

\section{SNP selection}

In our recent studies, ${ }^{14,26}$ we performed an extensive analysis of SNPs in $C F H$ and selected a total of 11 SNPs with $80 \%$ coverage of the gene. We genotyped all these SNPs in this study, including nine tagging SNPs (rs800292, rs10801555, rs10922096, rs10733086, rs10737680, rs11582939, rs2019727, rs1410996 and rs426736) from the $5^{\prime}$ to $3^{\prime}$ regions of $\mathrm{CFH}$ that were selected from phase 2 of the HapMap project $^{27}$ using the Tagger algorithm with an $r^{2}$ cutoff of 0.8 (minor allele frequency $>0.05$ ) and two important functional variants rs1061170 (p.Y402H) and rs460184 (p.V1197A) that were previously reported to be associated with agerelated macular degeneration and other human diseases. ${ }^{28,29}$ Detailed information of these selected SNPs is shown in our previous publications. ${ }^{14,26}$

\section{Genotyping}

Genomic DNA was isolated from whole blood using a Tiangen DNA isolation kit (Tiangen Biotech, Beijing, China). The 11 SNPs were detected using multiplex polymerase chain reaction and the SNaPshot assay, while details have been described in our previous work. ${ }^{14,26}$

\section{Psychiatric Genomics Consortium data analysis}

To further validate the association between the studied SNPs and schizophrenia, we extracted the schizophrenia genetic association data from the Psychiatric Genomics Consortium (PGC; http://www.broadinstitute.org/mpg/ricopili/) database $\mathrm{e}^{30}$ and reanalyzed the data set as an independent sample.

\section{Brain eQTL analysis for risk SNPs}

The brain eQTL analysis was performed using the brain eQTL database (http://caprica.genetics.kcl.ac.uk/BRAINEAC/), a large exon-specific eQTL data set covering 10 human brain regions. More detailed information can be found in the original study. ${ }^{31}$

\section{Statistical analysis}

Demographic data were analyzed using chi-squared or $t$-test as appropriate. For expression analyses, analysis of covariance (ANCOVA) was carried out with age, sex and smoking status as covariates controlled in the model to minimize the potential effect of these factors on the expression level of CFH messenger RNA. Hardy-Weinberg equilibrium testing and allele and genotype frequency analysis were conducted using SHEsis (http://analysis.bio-x.cn). ${ }^{32}$ The pairwise linkage disequilibrium (LD) analysis for all pairs of SNPs was applied to detect the inter-marker relationship in casecontrol samples. The LD blocks were identified using the solid spine of LD method, with extended spine if $D^{\prime}>0.5$ in Haploview (version 4.1). The possible genotype-phenotype correlation of $\mathrm{Y} 402 \mathrm{H}$ with schizophrenia symptoms was examined using ANCOVA by comparing the mean PANSS and RBANS scores of each genotype. Variables that affect symptom severity (that is, age, sex, education and duration of illness) were included as covariates. Statistical analyses were performed using SPSS 17.0 (SPSS Inc., Chicago, IL, USA). All $P$-values were two-tailed, and $P$-values $<0.05$ were considered statistically significant after Bonferroni correction.

\section{Results}

We extracted brain $\mathrm{CFH}$ expression data between schizophrenia patients and healthy controls from SZDB database. Table 1 showed that there is a significant association of hippocampal $C F H$ expression with schizophrenia $(P=0.017)$, whereas this significance did not survive after adjusting for false discovery rate $(P=0.105)$. However, patients with schizophrenia seem to have higher levels of $\mathrm{CFH}$ expression in hippocampus than controls (Figure 1).

Genotype distributions revealed no deviation from Hardy-Weinberg equilibrium in controls, except for rs460184, and we excluded this SNP from the following study. The genotype and allele frequencies of these CFH SNPs are presented in Table 2. There was a significant difference in allelic distribution of SNP rs1061170 $(\mathrm{Y} 402 \mathrm{H})$ between the case and control groups $(P=0.03)$. However, this significance did not remain after correcting for multiple testing ( $P=0.30$, after Bonferroni correction).

Table I CFH expression level in the brain between case and control groups

\begin{tabular}{|c|c|c|c|c|c|c|c|c|c|c|}
\hline \multirow[t]{2}{*}{ Gene } & \multirow[t]{2}{*}{ Probe } & \multicolumn{3}{|c|}{ Hippocampus } & \multicolumn{3}{|c|}{ Prefrontal cortex } & \multicolumn{3}{|l|}{ Stratum } \\
\hline & & Fold change & $P$-value ${ }^{a}$ & $P$-value ${ }^{b}$ & Fold change & $P$-value ${ }^{a}$ & $P$-value ${ }^{b}$ & Fold change & $P$-value ${ }^{a}$ & $P$-value ${ }^{b}$ \\
\hline CFH & 213800_at & 1.42 & 0.017 & 0.105 & 1.07 & 0.548 & 0.856 & 1.31 & 0.043 & 0.316 \\
\hline
\end{tabular}

Notes: Data from SZDB. ${ }^{\text {P }}$-values not corrected for multiple testing. ${ }^{b} P$-values adjusted after FDR correction.

Abbreviations: $\mathrm{CFH}$, complement factor H; FDR, false discovery rate; SZDB, A Database for Schizophrenia Genetic Research. 


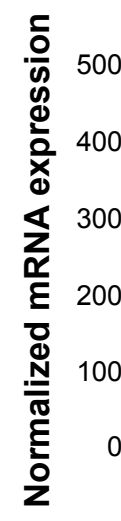

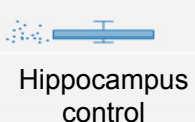
control
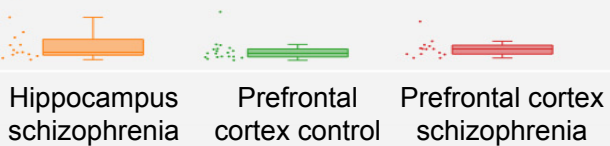

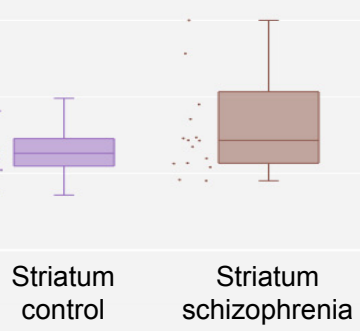

Figure I Differential expression of CFH in the brain between patients with schizophrenia and healthy controls.

Notes: Each bar represents the average level of CFH expression. Error bars represent the standard deviation of the mean value. Data were extracted from the SZDB (http://www.szdb.org/).

Abbreviations: $\mathrm{CFH}$, complement factor H; SZDB, A Database for Schizophrenia Genetic Research.

We further examined the genetic association between the 10 SNPs and schizophrenia in the PGC database. Although we did not find the data of rs1061170 in PGC database, Figure S1 showed that its tag SNP rs1061147 is not associated with schizophrenia in PGC GWAS $(P=0.273)$. Thus, none of the SNPs exhibited significant association with schizophrenia.
Analysis of pairwise LD showed three strong LDs between rs1061170 and rs10801555, rs 10922096 and rs2019727, as well as rs10733086 and rs10737680 (Figure S2). In view of the strong LDs, we performed a 2-SNP haplotype analysis, analyzing only those common haplotypes with at least $3 \%$ of frequency in either case or control samples ( $P$-values corresponding to the haplotypes are shown in

Table 2 Comparison of genotypic and allelic distributions of CFH variants between case and control groups

\begin{tabular}{|c|c|c|c|c|c|c|c|c|c|c|c|c|}
\hline \multirow{2}{*}{$\begin{array}{l}\text { SNP } \\
\text { rs800292 }\end{array}$} & \multirow{2}{*}{ Sample } & \multicolumn{3}{|c|}{ Genotype, n (\%) } & \multirow{2}{*}{$P$-value ${ }^{a}$} & \multicolumn{2}{|c|}{ Allele, n (\%) } & \multirow[t]{2}{*}{$P$-value ${ }^{a}$} & \multirow[t]{2}{*}{$P$-value ${ }^{b}$} & \multirow[t]{2}{*}{$P$-value } & \multirow[t]{2}{*}{$P$-value ${ }^{d}$} & \multirow{2}{*}{$\begin{array}{l}\text { Odds ratio } \\
(95 \% \mathrm{Cl})\end{array}$} \\
\hline & & $\mathrm{T} / \mathrm{T}$ & $\mathrm{T} / \mathrm{C}$ & $\mathrm{C} / \mathrm{C}$ & & $\mathrm{T}$ & C & & & & & \\
\hline & Cases & $135(15.4)$ & $45 \mathrm{I}(5 \mathrm{I} .4)$ & $292(33.3)$ & 0.86 & $72 \mid(4 I . \mid)$ & I,035 (58.9) & 0.89 & & 0.006 & 0.06 & $1.01(0.88-1.15)$ \\
\hline & Controls & $143(15.8)$ & $453(50.1)$ & $309(34.1)$ & & $739(40.8)$ & $\mathrm{I}, 07 \mathrm{I}(59.2)$ & & & & & \\
\hline \multirow[t]{3}{*}{ rs 1061170} & & $\mathrm{C} / \mathrm{C}$ & $\mathrm{C} / \mathrm{T}$ & $\mathrm{T} / \mathrm{T}$ & & C & $\mathrm{T}$ & & & & & \\
\hline & Cases & $5(0.6)$ & $107(12.2)$ & 766 & 0.09 & II (6.7) & $\mathrm{I}, 639(93.3)$ & 0.03 & 0.30 & $N / A$ & & $1.36(1.03-1.81)$ \\
\hline & Controls & $2(0.2)$ & $86(9.5)$ & $817(90.3)$ & & $90(5.0)$ & I,720 (95.0) & & & & & \\
\hline \multirow[t]{3}{*}{ rs 10801555} & & $\mathrm{~A} / \mathrm{A}$ & $A / G$ & $\mathrm{G} / \mathrm{G}$ & & A & $\mathrm{G}$ & & & & & \\
\hline & Cases & $3(0.3)$ & $105(12.0)$ & 770 & 0.24 & III (6.3) & $\mathrm{I}, 645(93.7)$ & 0.09 & & 0.244 & & $1.27(0.96-1.70)$ \\
\hline & Controls & $2(0.2)$ & $87(9.6)$ & $816(90.2)$ & & $91(5.0)$ & I,7I9 (95.0) & & & & & \\
\hline \multirow[t]{3}{*}{ rs 10922096} & & $\mathrm{~T} / \mathrm{T}$ & $T / C$ & $\mathrm{C} / \mathrm{C}$ & & $\mathrm{T}$ & C & & & & & \\
\hline & Cases & $16(1.8)$ & $219(24.9)$ & $643(73.2)$ & 0.59 & $25 I(14.3)$ & I,505 (85.7) & 0.32 & & 0.131 & & $0.99(0.91-1.33)$ \\
\hline & Controls & $13(1.4)$ & $212(23.4)$ & $680(75.1)$ & & $238(13.1)$ & $1,572(86.9)$ & & & & & \\
\hline \multirow[t]{3}{*}{ rs2019727 } & & $\mathrm{T} / \mathrm{T}$ & T/A & $\mathrm{A} / \mathrm{A}$ & & $\mathrm{T}$ & A & & & & & \\
\hline & Cases & $4(0.5)$ & $132(15.0)$ & $742(84.5)$ & 0.53 & I $40(8.0)$ & $1,616(92.0)$ & 0.52 & & 0.599 & & $0.93(0.73-1.17)$ \\
\hline & Controls & $8(0.9)$ & $139(15.4)$ & $758(83.8)$ & & $155(8.6)$ & I,655 (9l.4) & & & & & \\
\hline \multirow[t]{3}{*}{ rs 10733086} & & $\mathrm{~A} / \mathrm{A}$ & $\mathrm{A} / \mathrm{T}$ & $\mathrm{T} / \mathrm{T}$ & & A & $\mathrm{T}$ & & & & & \\
\hline & Cases & $6(0.7)$ & $137(15.6)$ & 735 & 0.57 & $149(8.5)$ & $\mathrm{I}, 607(91.5)$ & 0.31 & & 0.742 & & I.I $3(0.89-1.44)$ \\
\hline & Controls & $4(0.4)$ & $129(14.3)$ & $772(85.3)$ & & $137(7.6)$ & I,673 (92.4) & & & & & \\
\hline \multirow{3}{*}{ rs 10737680} & & $\mathrm{C} / \mathrm{C}$ & $\mathrm{C} / \mathrm{A}$ & $\mathrm{A} / \mathrm{A}$ & & C & $A$ & & & & & \\
\hline & Cases & I 48 (I6.9) & $448(51.0)$ & $282(32.1)$ & 0.75 & $744(42.4)$ & $1,012(57.6)$ & $0.8 \mathrm{I}$ & & 0.112 & & $0.98(0.86-1.12)$ \\
\hline & Controls & $163(18.0)$ & 448 (49.5) & $294(32.5)$ & & $774(42.8)$ & $1,036(57.2)$ & & & & & \\
\hline \multirow[t]{3}{*}{ rs1410996 } & & $\mathrm{T} / \mathrm{T}$ & $\mathrm{T} / \mathrm{C}$ & $\mathrm{C} / \mathrm{C}$ & & $\mathrm{T}$ & C & & & & & \\
\hline & Cases & $146(16.6)$ & 451 (5I.4) & $281(32.0)$ & 0.60 & $743(42.3)$ & $\mathrm{I}, 013(57.7)$ & 0.86 & & $N / A$ & & $0.99(0.87-1.13)$ \\
\hline & Controls & $163(18.0)$ & 445 (49.2) & $297(32.8)$ & & $771(42.6)$ & I,039 (57.4) & & & & & \\
\hline \multirow{3}{*}{ rsl|582939 } & & $\mathrm{T} / \mathrm{T}$ & $\mathrm{T} / \mathrm{C}$ & $\mathrm{C} / \mathrm{C}$ & & $\mathrm{T}$ & C & & & & & \\
\hline & Cases & $186(21.2)$ & $485(55.2)$ & $207(23.6)$ & 0.33 & $857(48.8)$ & $899(5 \mid .2)$ & 0.56 & & 0.106 & & $0.96(0.84-1.10)$ \\
\hline & Controls & $215(23.8)$ & $47 \mid(52.0)$ & $219(24.2)$ & & 901 (49.8) & $909(50.2)$ & & & & & \\
\hline \multirow[t]{3}{*}{ rs426736 } & & $\mathrm{C} / \mathrm{C}$ & $\mathrm{C} / \mathrm{T}$ & $\mathrm{T} / \mathrm{T}$ & & $\mathrm{C}$ & $\mathrm{T}$ & & & & & \\
\hline & Cases & $218(24.8)$ & $453(51.6)$ & $207(23.6)$ & 0.14 & $889(50.6)$ & $867(49.4)$ & 0.34 & & N/A & & $0.89(0.82-1.07)$ \\
\hline & Controls & $258(28.5)$ & 429 (47.4) & $218(24.1)$ & & $945(52.2)$ & $865(47.8)$ & & & & & \\
\hline
\end{tabular}

Notes: ${ }^{P}$-values not corrected for multiple testing. ${ }^{b} P$-values adjusted after Bonferroni correction. ${ }^{c} P$-values for $P G C$. ${ }^{d}$-values for $P G C$ after Bonferroni correction. Significant values are shown in bold.

Abbreviations: $\mathrm{CFH}$, complement factor H; SNP, single-nucleotide polymorphism; Cl, confidence interval; N/A, not applicable; PGC, Psychiatric Genomics Consortium. 
Table 3 Comparison of clinical characteristics among $\mathrm{Y} 402 \mathrm{H}$ genotypic groups in schizophrenia

\begin{tabular}{|c|c|c|c|c|c|}
\hline & $C / C+C / T(n=26)$ & $T / T(n=228)$ & $F$-value ${ }^{a}$ & $P$-value ${ }^{b}$ & $P$-value ${ }^{c}$ \\
\hline \multicolumn{6}{|l|}{ PANSS } \\
\hline Positive symptom & $10.54 \pm 3.12$ & $10.32 \pm 3.27$ & 0.21 & 0.88 & \\
\hline Negative symptom & $15.73 \pm 4.08$ & $12.01 \pm 3.72$ & 23.88 & 0.00 & 0.00 \\
\hline General psychopathology & $24.8 I \pm 4.46$ & $22.76 \pm 4.57$ & 4.79 & 0.03 & 0.30 \\
\hline Total score & $51.08 \pm 7.70$ & $45.10 \pm 8.93$ & 10.70 & 0.001 & \\
\hline \multicolumn{6}{|l|}{ RBANS } \\
\hline Immediate memory & $57.08 \pm 13.43$ & $60.60 \pm 11.79$ & 2.33 & 0.13 & \\
\hline Visuospatial skill & $58.04 \pm 7.58$ & $58.75 \pm 6.74$ & 0.39 & 0.54 & \\
\hline Language & $55.38 \pm 3.44$ & $55.53 \pm 4.95$ & 0.002 & 0.97 & \\
\hline Attention & $64.85 \pm 15.13$ & $71.39 \pm 19.00$ & 2.52 & 0.11 & \\
\hline Delayed memory & $61.38 \pm 13.77$ & $67.83 \pm 10.23$ & 8.27 & 0.004 & 0.04 \\
\hline Total score & $296.73 \pm 33.98$ & $314.10 \pm 35.96$ & 5.51 & 0.02 & 0.20 \\
\hline
\end{tabular}

Notes: Data presented as mean \pm SD. ${ }^{a} F$-values adjusted for age, sex, education and during of illness. ${ }^{b} P$-values not corrected for multiple testing. ${ }^{c} P$-values adjusted after Bonferroni correction.

Abbreviations: PANSS, Positive and Negative Syndrome Scale for Schizophrenia; RBANS, Repeatable Battery for the Assessment of Neuropsychological Status; SD, standard deviation.

Table S1). However, no significant difference was found for any haplotype.

We further examined the relationship between the $\mathrm{Y} 402 \mathrm{H}$ polymorphism and schizophrenia symptoms by comparing scores of the PANSS scale and RBANS with genotypes of the $\mathrm{Y} 402 \mathrm{H}$ polymorphism. Taking into consideration the low frequency of $\mathrm{C} / \mathrm{C}$ genotype in our sample, ANCOVA was carried out with the $\mathrm{Y} 402 \mathrm{H}$ genotypes $(\mathrm{C} / \mathrm{C}+\mathrm{C} / \mathrm{T}$ versus $\mathrm{T} / \mathrm{T})$ as the independent variables, the scores of PANSS scale and RBANS as the dependent variables and age, sex, years of education and duration of illness as the covariates. Table 3 showed significant differences in the scores of negative symptoms and delayed memory between the patients with $\mathrm{C}$ allele and those without $\mathrm{C}$ allele $(P<0.01$ and $P=0.04$ after Bonferroni correction, respectively).

To detect the role of $\mathrm{Y} 402 \mathrm{H}$ in hippocampal $\mathrm{CFH}$ expression, we performed an eQTL analysis. As shown in Figure 2, we observed a marginally significant association between the $\mathrm{Y} 402 \mathrm{H}$ polymorphism and $\mathrm{CFH}$ expression in the hippocampus $(P=0.051)$; however, this significance was lost after multiple testing correction $(P=0.51$, after Bonferroni correction).

\section{Discussion}

A recent cross-disorder genome-wide analysis showed that a broad set of common variants has cross-disorder effects for all the adult-onset disorders (MDD and schizophrenia and bipolar disorder). ${ }^{33}$ This group also calculated the genetic correlation of different psychiatric disorders using common SNPs and found that there was a moderate value $(0.47 \pm 0.06$ standard error [s.e.]) between MDD and schizophrenia. ${ }^{34}$ It is suggested that some similar brain pathology may be shared by the two psychiatric disorders. In the past decades, a body of literature has supported the implication

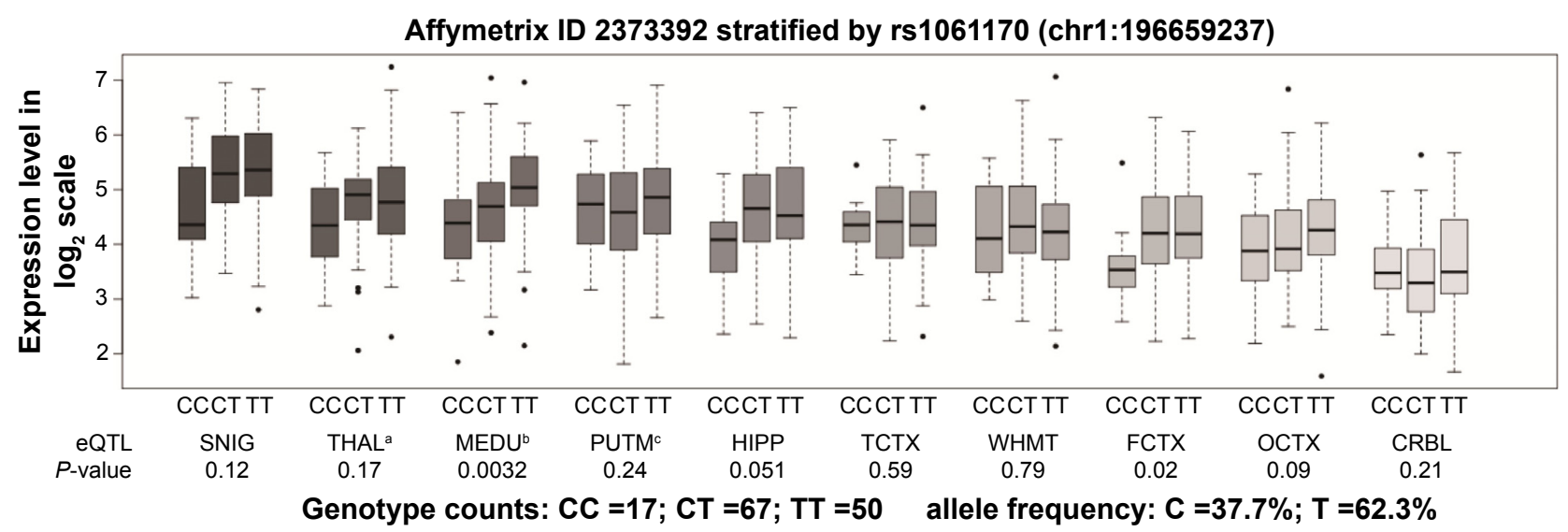

Figure 2 Association of rs 1061170 with CFH expression level in 10 brain regions (Affymetrix ID 2373392).

Notes: aAt the level of the lateral geniculate nucleus; 'bub-dissected from the medulla; ${ }^{c}$ at the level of the anterior commissure. Data were extracted from the BRAINEAC database (http://caprica.genetics.kcl.ac.uk/BRAINEAC/).

Abbreviations: BRAINEAC, The Brain eQTL Almanac; CFH, complement factor H; eQTL, expression quantitative trait locus; SNIG, substantia nigra; THAL, thalamus; MEDU, inferior olivary nucleus; PUTM, putamen; HIPP, hippocampus; TCTX, temporal cortex; WHMT, intralobular white matter; FCTX, frontal cortex; OCTX, occipital cortex; CRBL, cerebellar cortex. 
of immune alterations in MDD and schizophrenia. ${ }^{35}$ Our previous work indicated that $C F H$ plays a major role in the development of MDD. ${ }^{14}$ On this premise, we attempted to investigate the role of $C F H$ in schizophrenia. We investigated 11 SNPs within $C F H$ and carried out the PGC analysis for further validation. Although the data of rs 1061170 (Y402H) were not found in the PGC database, its tag SNP rs1061147 that has perfect LD $\left(r^{2}=1.0\right)$ with $\mathrm{rs} 1061170$ shows no significant association with schizophrenia in PGC GWAS. The frequency of A allele of rs1061147 is similar to that of $\mathrm{C}$ allele of rs 1061170 either in Caucasian (36\%) or in Han Chinese (7\%) populations. Therefore, our results implied that there is no significant association of $\mathrm{CFH}$ with schizophrenia in either Chinese Han or Caucasian populations. However, we found that hippocampal $\mathrm{CFH}$ expression may be enriched in patients with schizophrenia than healthy controls through an eQTL analysis using the SZDB database.

$\mathrm{CFH}$ is a major inhibitor of the alternative complement pathway, which regulates complement activation in tissue inflammation during degeneration. ${ }^{36}$ Previous literature has reported that increased serum $\mathrm{CFH}$ level is associated with Alzheimer's disease, a neurodegenerative disorder. ${ }^{37,38}$ Thus, increased CFH expression may be implicated with the development of neurodegeneration. On the other side, it has been well documented that the largest magnitude of subcortical brain volume abnormalities in schizophrenia is in the hippocampus, which can be seen in both the early and chronic stages of this disorder. ${ }^{39,40}$ Hippocampus is hypothesized to underlie the neuropsychological deficits and symptoms observed in schizophrenia. ${ }^{41,42}$ Thereby, the role of $\mathrm{CFH}$ in schizophrenia could not be excluded, even though we did not detect any association of $\mathrm{CFH}$ with schizophrenia at molecular level. The finding that hippocampal $C F H$ expression alters in schizophrenia implied that $\mathrm{CFH}$ may be involved in certain specific symptoms of this disorder. The $\mathrm{Y} 402 \mathrm{H}$ polymorphism is a non-synonymous SNP and is of particular interest because it is located within the region of short consensus repeat domains 7 binding heparin and C-reactive protein. ${ }^{43}$ The base transition of thymine to cytosine occurs in the exon 9 of the gene and leads to a tyrosine-histidine substitution in the protein. ${ }^{44}$ Previous studies demonstrated that this variant exerts allelic differences on the binding affinity to C-reactive proteins, with the risk allele showing reduced affinity. ${ }^{45}$ In doing so, this could influence complement activation, host immune status and inflammation process and hence account for $\sim 17 \%$ of age-related macular degeneration liability. ${ }^{46}$ Hence, we further examined whether $\mathrm{Y} 402 \mathrm{H}$ polymorphism is associated with clinical dimensions of schizophrenia.

In general, patients with schizophrenia performed worse in cognitive function than healthy controls in almost all the cognitive domains. ${ }^{47,48}$ Among the case group, we observed a positive association of $\mathrm{Y} 402 \mathrm{H}$ polymorphism with the severity of negative symptoms and delayed memory. Negative symptoms are deficits of normal emotional responses, including avolition, affective flattening and social withdrawal. ${ }^{49}$ There is considerable conceptual overlap between the negative symptoms and cognitive dysfunction. ${ }^{50}$ The postmortem studies are consistent with the neuroimaging findings showing an association of altered structure and function of hippocampus with schizophrenia. ${ }^{51}$ There is evidence from functional magnetic resonance imaging study showing an association of hippocampal neural activity with amygdala activity and emotional memory, ${ }^{52}$ suggesting an involvement of hippocampus in emotional processing. A recent functional magnetic resonance imaging study showed hippocampal hypoactivity in patients with schizophrenia during facial emotional processing tasks. ${ }^{53}$ On the other side, the hippocampus has been well established to be necessary for learning and memory. ${ }^{54} \mathrm{~A}$ line of MRI scans have indicated that hippocampus plays a critical role in cognitive dysfunction in schizophrenia. ${ }^{55}$ Therefore, hippocampus is likely to be a crucial brain region in the development of negative symptoms and cognitive dysfunction in schizophrenia. To detect the association of $\mathrm{Y} 402 \mathrm{H}$ polymorphism and $\mathrm{CFH}$ expression in hippocampus, we performed an eQTL analysis. Our results implied that the $\mathrm{Y} 402 \mathrm{H}$ polymorphism has a possible modulatory effect on $\mathrm{CFH}$ expression in hippocampus. As such, these findings suggested that $\mathrm{Y} 402 \mathrm{H}$ polymorphism may give risk to the alternation of $\mathrm{CFH}$ expression in hippocampus and influence the severity of negative symptoms and cognitive dysfunction in schizophrenia. We noticed in SZDB database that patients with schizophrenia had higher levels of hippocampal $\mathrm{CFH}$ expression than controls. However, in the brain eQTL analysis for risk SNP, hippocampal $\mathrm{CFH}$ expression seemed lower in individuals with the risk $\mathrm{C} / \mathrm{C}$ genotype than those with $\mathrm{C} / \mathrm{T}$ or $\mathrm{T} / \mathrm{T}$ genotypes. The counterintuitive results may be caused by small sample size and different ethnic origins. Therefore, further investigations are warranted to address this issue.

When interpreting the results of this study, we would be remiss in not noting some limitations. First, cross-sectional association studies always have the potential for population 
stratification. Although the subjects were all of Han Chinese origin and collected from Eastern China, we could not fully exclude the possibility of a population structure effect in our sample. Second, this study details an exploratory study performed on a subset of the general Chinese Han population. The sample size is modest and precludes us from making any definitive statements on the associations between $\mathrm{CFH}$ and schizophrenia in Han Chinese. Third, all the patients had received antipsychotic treatment and maintained stable conditions for $>6$ months prior to this study. It is known that antipsychotic treatment would bias symptomatology, and therefore, we could not completely conclude that $\mathrm{CFH}$ is associated with negative symptoms and cognitive dysfunction in schizophrenia. Accordingly, our findings should be considered only preliminary and exploratory. Further investigations need to validate our results in independent populations and more fully explain any potential relationship or lack thereof.

\section{Conclusion}

We performed a comprehensive analysis for the association between $\mathrm{CFH}$ and schizophrenia in Han Chinese. Our findings provided suggestive evidence for $C F H$ 's role in the development of negative symptoms and cognitive dysfunction in schizophrenia. Further investigations are required to evaluate this association in a larger and independent sample across various ethnicities.

\section{Acknowledgments}

The authors are deeply grateful to all participants. This work was supported by the National Natural Science Foundation of China (81471358 and 81671326), the Shanghai Science and Technology Commission Foundation (14411969000), the Shanghai Municipal Education Commission - Gaofeng Clinical Medicine Grant Support (20152530), the Shanghai Municipal Commission of Health and Family Planning Foundation (201540029) and the Shanghai Mental Health Center Foundation (2014-FX-03).

\section{Author contributions}

All authors contributed toward data analysis, drafting and critically revising the paper, gave final approval of the version to be published, and agree to be accountable for all aspects of the work.

\section{Disclosure}

The authors report no conflicts of interest in this work.

\section{References}

1. Dhindsa RS, Goldstein DB. Schizophrenia: from genetics to physiology at last. Nature. 2016;530(7589):162-163.

2. Brown AS, Derkits EJ. Prenatal infection and schizophrenia: a review of epidemiologic and translational studies. Am J Psychiatry. 2010;167(3): 261-280.

3. Benros ME, Nielsen PR, Nordentoft M, Eaton WW, Dalton SO, Mortensen PB. Autoimmune diseases and severe infections as risk factors for schizophrenia: a 30-year population-based register study. Am J Psychiatry. 2011;168(12):1303-1310.

4. Goldman AL, Pezawas L, Mattay VS, et al. Heritability of brain morphology related to schizophrenia: a large-scale automated magnetic resonance imaging segmentation study. Biol Psychiatry. 2008;63(5): 475-483.

5. Sullivan PF, Kendler KS, Neale MC. Schizophrenia as a complex trait: evidence from a meta-analysis of twin studies. Arch Gen Psychiatry. 2003;60(12):1187-1192.

6. Purcell SM, Wray NR, Stone JL, et al. Common polygenic variation contributes to risk of schizophrenia and bipolar disorder. Nature. 2009; 460(7256):748-752.

7. Stefansson H, Ophoff RA, Steinberg S, et al. Common variants conferring risk of schizophrenia. Nature. 2009;460(7256):744-747.

8. Shi J, Levinson DF, Duan J, et al. Common variants on chromosome 6p22.1 are associated with schizophrenia. Nature. 2009;460(7256): $753-757$.

9. Ikeda M, Aleksic B, Kinoshita Y, et al. Genome-wide association study of schizophrenia in a Japanese population. Biol Psychiatry. 2011; 69(5):472-478.

10. Zhang Y, Lu TL, Yan H, et al. Replication of association between schizophrenia and chromosome 6p21-6p22.1 polymorphisms in Chinese Han population. PLoS One. 2013;8(2):e56732.

11. Yue WH, Wang HF, Sun LD, et al. Genome-wide association study identifies a susceptibility locus for schizophrenia in Han Chinese at 11p11.2. Nat Genet. 2011;43(12):1228-1284.

12. Sekar A, Bialas AR, de Rivera H, et al. Schizophrenia risk from complex variation of complement component 4. Nature. 2016;530(7589): $177-183$.

13. Ripoche J, Day AJ, Harris TJ, Sim RB. The complete amino acid sequence of human complement factor H. Biochem J. 1988;249(2):593-602.

14. Zhang C, Zhang DF, Wu ZG, et al. Complement factor H and susceptibility to major depressive disorder in Han Chinese. Br J Psychiatry. 2016;208(5):446-452.

15. Craddock N, Forty L. Genetics of affective (mood) disorders. Eur J Hum Genet. 2006;14(6):660-668.

16. Wang M, Chen J, He K, et al. The NVL gene confers risk for both major depressive disorder and schizophrenia in the Han Chinese population. Prog Neuropsychopharmacol Biol Psychiatry. 2015;62:7-13.

17. Chen J, Wang M, Waheed Khan RA, et al. The GSK3B gene confers risk for both major depressive disorder and schizophrenia in the Han Chinese population. J Affect Disord. 2015;185:149-155.

18. Papiol S, Malzahn D, Kastner A, et al. Dissociation of accumulated genetic risk and disease severity in patients with schizophrenia. Transl Psychiatry. 2011;1:e45.

19. Zhang XY, Chen DC, Tan YL, et al. BDNF polymorphisms are associated with schizophrenia onset and positive symptoms. Schizophr Res. 2016;170(1):41-47.

20. Cai J, Zhu Y, Zhang W, Wang Y, Zhang C. Comprehensive family therapy: an effective approach for cognitive rehabilitation in schizophrenia. Neuropsychiatr Dis Treat. 2015;11:1247-1253.

21. Zhang C, Li Z, Shao Y, et al. Association study of tryptophan hydroxylase-2 gene in schizophrenia and its clinical features in Chinese Han population. J Mol Neurosci. 2011;43(3):406-411.

22. Kay SR, Fiszbein A, Opler LA. The positive and negative syndrome scale (PANSS) for schizophrenia. Schizophr Bull. 1987;13(2): 261-276. 
23. Randolph C, Tierney MC, Mohr E, Chase TN. The repeatable battery for the assessment of neuropsychological status (RBANS): preliminary clinical validity. J Clin Exp Neuropsychol. 1998;20(3):310-319.

24. Fornito A, Bullmore ET. Reconciling abnormalities of brain network structure and function in schizophrenia. Curr Opin Neurobiol. 2015; 30:44-50.

25. Wu Y, Yao YG, Luo XJ. SZDB: a database for schizophrenia genetic research. Schizophr Bull. Epub 2016 Jul 22. doi:10.1093/schbul/ sbw102.

26. Zhang DF, Wang D, Li YY, Yao YG. Mapping genetic variants in the CFH gene for association with leprosy in Han Chinese. Genes Immun. 2014;15(7):506-510.

27. International HapMap Consortium. The International HapMap Project. Nature. 2003;426(6968):789-796.

28. Chen H, Liu K, Chen LJ, Hou P, Chen W, Pang CP. Genetic associations in polypoidal choroidal vasculopathy: a systematic review and meta-analysis. Mol Vis. 2012;18:816-829.

29. Huang L, Li Y, Guo S, et al. Different hereditary contribution of the CFH gene between polypoidal choroidal vasculopathy and age-related macular degeneration in Chinese Han People. Invest Ophthalmol Vis Sci. 2014;55(4):2534-2538.

30. Schizophrenia Psychiatric Genome-Wide Association Study (GWAS) Consortium. Genome-wide association study identifies five new schizophrenia loci. Nat Genet. 2011;43(10):969-976.

31. Ramasamy A, Trabzuni D, Guelfi S, et al. Genetic variability in the regulation of gene expression in ten regions of the human brain. Nat Neurosci. 2014;17(10):1418-1428.

32. Shi YY, He L. SHEsis, a powerful software platform for analyses of linkage disequilibrium, haplotype construction, and genetic association at polymorphism loci. Cell Res. 2005;15(2):97-98.

33. Cross-Disorder Group of the Psychiatric Genomics Consortium. Identification of risk loci with shared effects on five major psychiatric disorders: a genome-wide analysis. Lancet. 2013;381(9875):1371-1379.

34. Lee SH, Ripke S, Neale BM, et al; International Inflammatory Bowel Disease Genetics Consortium (IIBDGC). Genetic relationship between five psychiatric disorders estimated from genome-wide SNPs. Nat Genet. 2013;45(9):984-994.

35. Lisboa SF, Gomes FV, Guimaraes FS, Campos AC. Microglial cells as a link between cannabinoids and the immune hypothesis of psychiatric disorders. Front Neurol. 2016;7:5.

36. Langford-Smith A, Keenan TD, Clark SJ, Bishop PN, Day AJ. The role of complement in age-related macular degeneration: heparan sulphate, a ZIP code for complement factor H? J Innate Immun. 2014; 6(4):407-416.

37. Hye A, Lynham S, Thambisetty M, et al. Proteome-based plasma biomarkers for Alzheimer's disease. Brain. 2006;129(pt 11):3042-3050.

38. Hye A, Riddoch-Contreras J, Baird AL, et al. Plasma proteins predict conversion to dementia from prodromal disease. Alzheimers Dement. 2014;10(6):799.e-807.e.

39. Adriano F, Caltagirone C, Spalletta G. Hippocampal volume reduction in first-episode and chronic schizophrenia: a review and meta-analysis. Neuroscientist. 2012;18(2):180-200.
40. van Erp TG, Hibar DP, Rasmussen JM, et al. Subcortical brain volume abnormalities in 2028 individuals with schizophrenia and 2540 healthy controls via the ENIGMA consortium. Mol Psychiatry. 2016;21(4):547-553.

41. Hýža M, Kuhn M, Češková E, Ustohal L, Kašpárek T. Hippocampal volume in first-episode schizophrenia and longitudinal course of the illness. World J Biol Psychiatry. 2016;17(6):429-438.

42. Ho NF, Iglesias JE, Sum MY, et al. Progression from selective to general involvement of hippocampal subfields in schizophrenia. Mol Psychiatry. 2017;22(1):142-152.

43. Klein RJ, Zeiss C, Chew EY, et al. Complement factor $\mathrm{H}$ polymorphism in age-related macular degeneration. Science. 2005;308(5720): 385-389.

44. Rodriguez de Cordoba S, Esparza-Gordillo J, Goicoechea de Jorge E, Lopez-Trascasa M, Sanchez-Corral P. The human complement factor H: functional roles, genetic variations and disease associations. Mol Immunol. 2004;41(4):355-367.

45. Chen H, Yu KD, Xu GZ. Association between variant Y402H in age-related macular degeneration (AMD) susceptibility gene CFH and treatment response of AMD: a meta-analysis. PLoS One. 2012;7(8):e42464.

46. Raychaudhuri S, Iartchouk $\mathrm{O}$, Chin $\mathrm{K}$, et al. A rare penetrant mutation in $\mathrm{CFH}$ confers high risk of age-related macular degeneration. Nat Genet. 2011;43(12):1232-1236.

47. Han M, Huang XF, Chen DC, et al. Gender differences in cognitive function of patients with chronic schizophrenia. Prog Neuropsychopharmacol Biol Psychiatry. 2012;39(2):358-363.

48. Zhang XY, Chen DC, Xiu MH, et al. Cognitive and serum BDNF correlates of BDNF Val66Met gene polymorphism in patients with schizophrenia and normal controls. Hum Genet. 2012;131(7):1187-1195.

49. Kirkpatrick B, Fenton WS, Carpenter WT Jr, Marder SR. The NIMHMATRICS consensus statement on negative symptoms. Schizophr Bull. 2006;32(2):214-219.

50. Fervaha G, Takeuchi H, Foussias G, Agid O, Remington G. Using poverty of speech as a case study to explore the overlap between negative symptoms and cognitive dysfunction. Schizophr Res. 2016;176(2-3): 411-416.

51. Heckers S, Konradi C. GABAergic mechanisms of hippocampal hyperactivity in schizophrenia. Schizophr Res. 2015;167(1-3):4-11.

52. Phelps EA. Human emotion and memory: interactions of the amygdala and hippocampal complex. Curr Opin Neurobiol. 2004; 14(2):198-202.

53. Ji E, Weickert CS, Lenroot R, et al. Adjunctive selective estrogen receptor modulator increases neural activity in the hippocampus and inferior frontal gyrus during emotional face recognition in schizophrenia. Transl Psychiatry. 2016;6:e795.

54. Zhang C, Fang Y, Xu L. Glutamate receptor 1 phosphorylation at serine 845 contributes to the therapeutic effect of olanzapine on schizophrenialike cognitive impairments. Schizophr Res. 2014;159(2-3):376-384.

55. Koolschijn PC, van Haren NE, Cahn W, et al. Hippocampal volume change in schizophrenia. J Clin Psychiatry. 2010;71(6):737-744. 


\section{Supplementary materials}

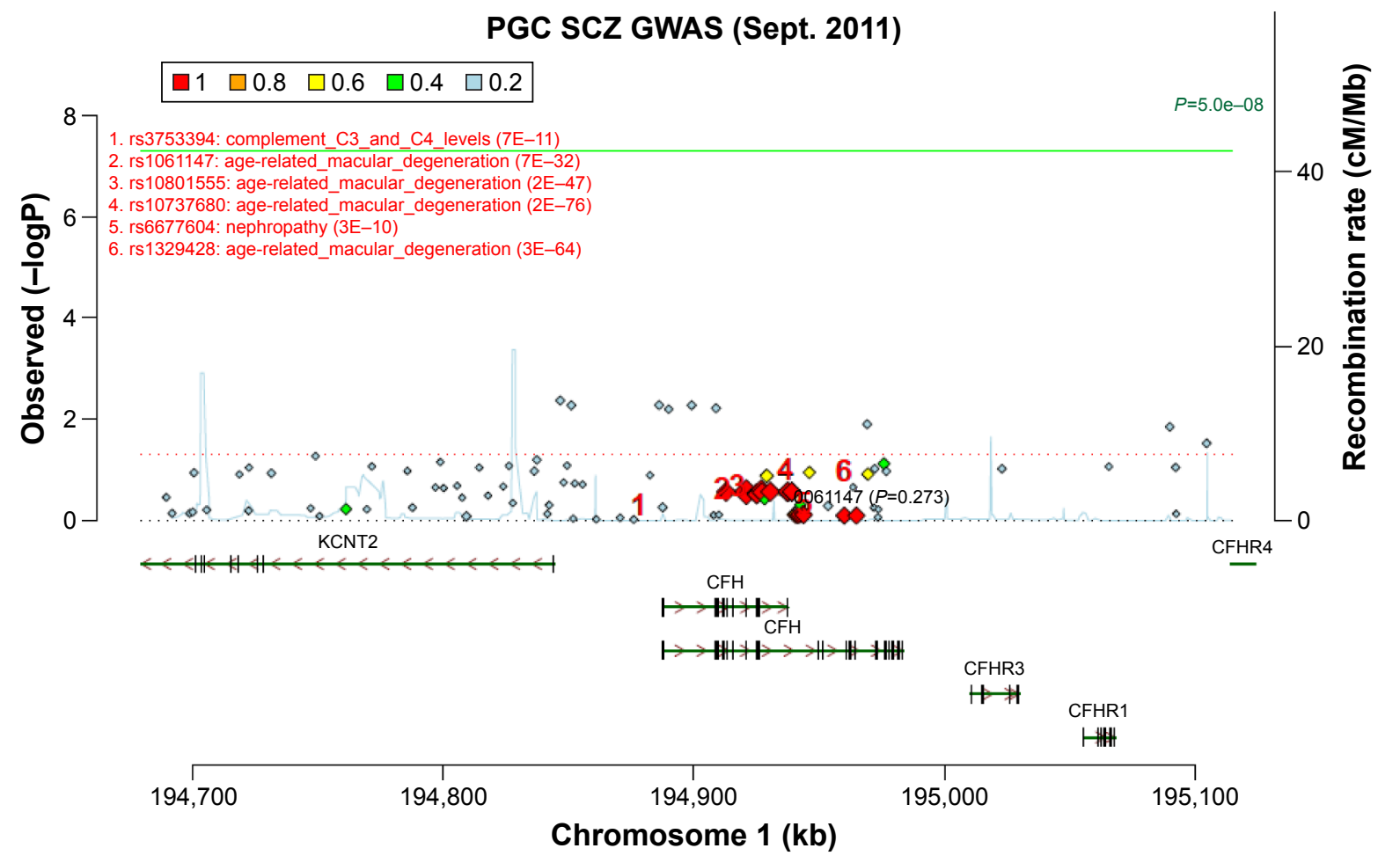

Figure SI Association of rs 106 I 47 with schizophrenia.

Note: Data from the Psychiatric Genomics Consortium (PGC; http://www.broadinstitute.org/mpg/ricopili/) database.

Abbreviations: PGC, Psychiatric Genomics Consortium; SCZ, schizophrenia; GWAS, genome-wide association studies; Sept., September.
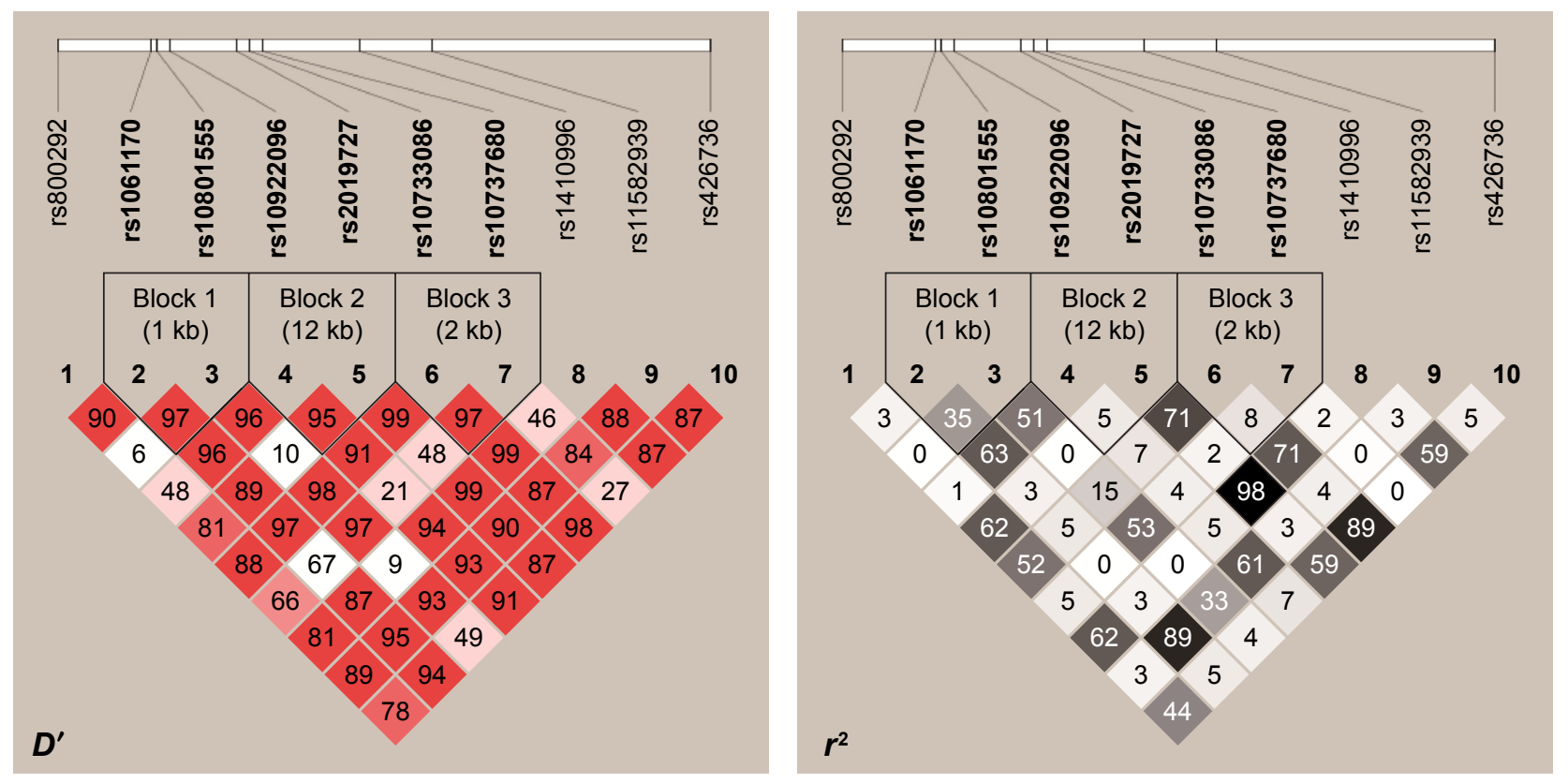

Figure S2 LD plot consisting of I0 SNPs at the CFH gene and its region plot.

Notes: Pairwise LD was computed for all possible combinations of the 10 SNPs using the values of $D^{\prime}$ and $r^{2}$. The individual square showed the $100 \times D^{\prime}$ (or $r^{2}$ ) value for each SNP pair. SNP rs460I84 was not included due to the deviation from HWE.

Abbreviations: LD, linkage disequilibrium; SNP, single-nucleotide polymorphism; CFH, complement factor H; HWE, Hardy-Weinberg equilibrium. 
Table SI Results of the pairwise haplotype test of the case and control groups

\begin{tabular}{|c|c|c|c|c|}
\hline \multirow[t]{2}{*}{ Haplotype $^{a}$} & \multicolumn{2}{|c|}{ Frequency (\%) } & \multirow[t]{2}{*}{$P$-values ${ }^{b}$} & \multirow[t]{2}{*}{$P$-values } \\
\hline & Case & Control & & \\
\hline \multicolumn{5}{|c|}{ rsI06II70-rsI080I555 } \\
\hline T-G & 85.7 & 86.6 & 0.42 & \\
\hline T-A & 8.0 & 8.4 & 0.69 & \\
\hline C-A & 6.3 & 4.8 & 0.05 & 0.18 \\
\hline \multicolumn{5}{|c|}{ rs10922096-rs2019727 } \\
\hline C-A & 49.2 & 49.9 & 0.68 & \\
\hline C-T & 42.3 & 42.5 & 0.89 & \\
\hline T-A & 8.4 & 7.3 & 0.23 & \\
\hline \multicolumn{5}{|c|}{ rs10733086-rs10737680 } \\
\hline A-A & 48.8 & 49.6 & 0.61 & \\
\hline T-A & 43.3 & 41.8 & 0.38 & \\
\hline $\mathrm{T}-\mathrm{C}$ & 7.9 & 8.4 & 0.60 & \\
\hline
\end{tabular}

Notes: a Haplotypes with frequency $<0.03$ were ignored in analysis. ${ }^{\mathrm{T} T h e} P$-values for single haplotype test, $d f=I$, not corrected for multiple test. ${ }^{c}$-values adjusted after 10,000 permutations. Significance is presented in bold.

\section{Publish your work in this journal}

Neuropsychiatric Disease and Treatment is an international, peerreviewed journal of clinical therapeutics and pharmacology focusing on concise rapid reporting of clinical or pre-clinical studies on a range of neuropsychiatric and neurological disorders. This journal is indexed on PubMed Central, the 'PsycINFO' database and CAS, and is the official journal of The International Neuropsychiatric Association (INA). The manuscript management system is completely online and includes a very quick and fair peer-review system, which is all easy to use. Visit http://www.dovepress.com/testimonials.php to read real quotes from published authors.

\footnotetext{
Submit your manuscript here: http://www.dovepress.com/neuropsychiatric-disease-and-treatment-journal
} 